These authors give a mathematical definition connected to the unimodular $n \times n$ matrices of finite group associated to a $Z$ class.

Our definition is connected to the geometry and the splitting up of the metric tensor of a crystal cell and to the bases of the irreducible representations of the holohedry of the crystal family. For these reasons, the letter ' $g$ ' is the abbreviation of 'geometrical'; ' $Z$ ' is the group of positive or negative integers.

We suggested this definition:

Let $x, y, z, t, u, v, \ldots$ be the $n$ translation operators corresponding to a basis of a primitive Bravais cell of a crystal family of the $n$-dimensional space $E^{n}$. This family is said to be 'geometrically $Z$-irreducible' ( $\mathrm{g} Z$-irr.) if all these operators belong to the same irreducible representation with integer entries of the character table of its holohedry. If this property is not verified, the crystal family is said to be 'geometrically $Z$-reducible' (gZ-red.); in this case, the metric tensor can be split into two or more parts or, in other words, the cell of the crystal family is the orthogonal product of two or more cells belonging to two or more orthogonal subspaces of space $E^{n}$. Now, we give two simple examples:

The rectangular family of space $E^{2}$ is a $\mathrm{g} Z$ reducible family, the WPV symbol of the holohedry is $m \perp m$, the construction of the cell is explained as the rectangular product of two unequal segments. It is easy to see that the two operators $x$ and $y$ belong to two different irreducible representations of the character table of this holohedry.

The oblic family and the square family are gZ-irr. crystal families of space $E^{2}$. Indeed, the two operators $x$ and $y$ belong to the same irreducible representation of dimension 1 (which is not the identity representation) for the oblic family and to the same irreducible representation of dimension 2, for the square family. If we consider the metric tensor of these two cells, we notice that it is impossible to split them into two parts.

\section{References}

Brown, H., Bülow, R., Neubüser, J., WondratscheK, H. \& Zassenhaus, H. (1978). Crystallographic Groups of FourDimensional Space. Wiley: Interscience.

Phan, T. \& Veysseyre, R. (1994). Acta Cryst. A50, 438-444.

Phan, T., Veysseyre, R. \& Weigel, D. (1991). Acta Cryst. A47, 549-553.

Veysseyre, R. \& Weigel, D. (1989). Acta Cryst. A45, 187-193.

Veysseyre, R., Weigel, D. \& Phan, T. (1993). Acta Cryst. A49, 481-486.

Weigel, D., Phan, T. \& Veysseyre, R. (1987). Acta Cryst. A43, 294-304.

Weigel, D. \& Veysseyre, R. (1991). C. R. Acad. Sci. 313, Sér. II, 481-486.

Acta Cryst. (1994). A50, 450-455

\title{
The Centrosymmetric-Noncentrosymmetric Ambiguity: Some More Examples
}

\author{
By RICHARD E. MARSH \\ The Beckman Institute, California Institute of Technology, Pasadena, California 91125, USA*
}

(Received 16 June 1992; accepted 17 November 1993)

\begin{abstract}
Four more examples are provided to emphasize the extreme difficulty in deciding, by diffraction methods, whether a crystal structure is centrosymmetric or only approximately so. In these examples, earlier workers described and refined structures in noncentrosymmetric space groups; refinements in the corresponding centrosymmetric space groups, based on the original data, lead to improved results. In one case, apparent violations of systematic absences seem to preclude the centrosymmetric description; however, other evidence - in particular, improved agreement for the very weak reflections (which are the most sensitive to the centrosymmetric-non-

* Contribution no. 8375 .
\end{abstract}

(C) 1994 International Union of Crystallography

Printed in Great Britain - all rights reserved centrosymmetric ambiguity) - suggest that the spacegroup violations might be spurious. In any event, the moral is clear: extreme caution is needed when attempting to derive a noncentrosymmetric description of a closely centrosymmetric structure.

\section{Introduction}

For a number of years, I have been interested in the problem of attempting to decide, by means of X-ray diffraction alone, whether a particular crystal structure is centrosymmetric or only approximately so. As has been noted often, perhaps beginning with Ermer \& Dunitz (1970), the first small deviation from centrosymmetry cannot be detected by normal diffraction methods since the centrosymmetric model ISSN 0108-7673 (C) 1994 
represents an exact singularity in any solution process: the derivatives of all diffraction intensities relative to the antisymmetric deviations are identically zero. As the deviations become larger, the singularities become near-singularities, with large correlations between the various 'antisymmetric' parameters - the parameters describing the deviations from centrosymmetry (as opposed to those describing the average centrosymmetric structure, for which the correlations are normal). Because of these large correlations, convergence to a noncentrosymmetric solution may be hard to achieve; but even if convergence can be achieved - perhaps by applying damping factors or by an eigenvalue decomposition procedure - one must worry as to whether the correct solution has been found: with respect to the parameters describing the antisymmetric distortions, the minimum in the least-squares residual is a very broad one that might well contain numerous small local minima that are very sensitive to the weighting procedure and to systematic errors. One must then ask two questions: (1) Are the minima real and not the result of inaccurate data or unreliable weights? (2) If they are real, has the single correct minimum been found? If the answer to both questions is not 'yes' - if one cannot be sure that the structure is noncentrosymmetric and that the derived pattern of antisymmetric distortions is the correct pattern - it seems prudent to opt for the average centrosymmetric description, tacitly acknowledging that small antisymmetric distortions may be present but that they cannot be uniquely determined from the diffraction evidence (Marsh, 1986).

I have encountered a number of additional examples in which authors have described nearly centrosymmetric structures in noncentrosymmetric space groups, presuming that they have found the correct pattern of small antisymmetric distortions. In all these cases, it appears as though better results an improved $R$ value or more reasonable geometry or both - can be obtained in the centrosymmetric space groups. Details follow.

\section{Synthetic perryite}

The structure of this compound, whose composition as determined by electron probe microanalysis was reported as $\left(\mathrm{Ni}_{0.97} \mathrm{Fe}_{0.03}\right)_{8}\left(\mathrm{Si}_{0.79} \mathrm{P}_{0.21}\right)_{3}$ (Okada, Kobayashi, Ito \& Sakurai, 1991; hereinafter referred to as OKIS), was described in space group $R 3 c$ [hexagonal axes: $a=6.640(2), c=37.982(7) \AA, Z=$ 12] and refined to an $R$ of 0.027 for 333 reflections with $F_{o} \geq 3 \sigma\left(F_{o}\right)$. Space group $R \overline{3} c$ seems a far better choice: refinement in $R \overline{3} c$, based on the $333 F_{o}$ values in SUP 53898, led to a final $R$ of 0.023 and to considerably smaller e.s.d.'s than reported by OKIS.
Table 1. Perryite coordinates, space group $R \overline{3} c$

$x, y$ and $z$ are multiplied by $10^{5}$ and $U_{\text {eq }}$ is multiplied by $10^{4}$.

\begin{tabular}{lcccc}
\multicolumn{5}{c}{$U_{\text {eq }}=\frac{1}{3} \sum_{i} \sum_{j} U_{i j} a_{i}{ }^{*} a_{j}{ }^{*} \mathbf{a}_{i} \cdot \mathbf{a}_{j}}$. \\
$\mathrm{Ni}(\mathrm{Fe})(1)$ & $33167(13)$ & $41760(13)$ & $5226(2)$ & $U_{\text {eq }}\left(\AA^{2}\right)$ \\
$\mathrm{Ni}(\mathrm{Fe})(2)$ & $33764(13)$ & $3347(13)$ & $1744(2)$ & $69(1)$ \\
$\mathrm{Ni}(\mathrm{Fe})(3)$ & 0 & 0 & $21863(3)$ & $56(1)$ \\
$\mathrm{Ni}(\mathrm{Fe})(4)$ & 0 & 0 & $5959(3)$ & $56(1)$ \\
$\mathrm{Si}(\mathrm{P})(1)$ & $-31572(33)$ & 0 & 25000 & $56(2)$ \\
$\mathrm{Si}(\mathrm{P})(2)$ & 0 & 0 & $15823(6)$ & $43(2)$ \\
$\mathrm{Si}(\mathrm{P})(3)$ & 0 & 0 & 0 & $22(2)$
\end{tabular}

Table 2. Perryite anisotropic coefficients $\left(\AA^{2}\right)$, space group $R \overline{3} c$

$U_{i j}$ values have been multiplied by $10^{4}$. The form of the displacement factor is $\exp \left[-2 \pi^{2}\left(U_{11} h^{2} a^{* 2}+U_{22} k^{2} b^{* 2}+U_{33} l^{2} c^{* 2}+\right.\right.$ $\left.\left.2 U_{12} h k a^{*} b^{*}+2 U_{13} h l a^{*} c^{*}+2 U_{23} k l b^{*} c^{*}\right)\right]$.

\begin{tabular}{lcccccc} 
& $U_{11}$ & $U_{22}$ & \multicolumn{1}{c}{$U_{33}$} & $U_{12}$ & \multicolumn{1}{c}{$U_{13}$} & $U_{23}$ \\
$\mathrm{Ni}(\mathrm{Fe})(1)$ & $66(4)$ & $114(4)$ & $1(3)$ & $26(3)$ & $5(2)$ & $7(3)$ \\
$\mathrm{Ni}(\mathrm{Fe})(2)$ & $60(4)$ & $74(4)$ & $-2(3)$ & $26(3)$ & $-11(2)$ & $14(2)$ \\
$\mathrm{Ni}(\mathrm{Fe})(3)$ & $86(4)$ & $86(4)$ & $-3(5)$ & $43(2)$ & 0 & 0 \\
$\mathrm{Ni}(\mathrm{Fe})(4)$ & $86(4)$ & $86(4)$ & $-4(5)$ & $43(2)$ & 0 & 0 \\
$\mathrm{Si}(\mathrm{P})(1)$ & $79(8)$ & $81(11)$ & $7(8)$ & $40(5)$ & 0 & 0 \\
$\mathrm{Si}(\mathrm{P})(2)$ & $77(7)$ & $77(7)$ & $-26(10)$ & $38(4)$ & 0 & 0 \\
$\mathrm{Si}(\mathrm{P})(3)$ & $54(10)$ & $54(10)$ & $-41(13)$ & $27(5)$ & 0 & 0
\end{tabular}

The final $R \overline{3} c$ coordinates are given in Table 1 and the anisotropic coefficients $U_{i j}$ are given in Table 2.

A few comments on the $R \overline{3} c$ refinement are in order: (1) All the $U_{33}$ terms became unrealistically small or marginally negative (Table 2 ). The sa ne general phenomenon resulted from the $R 3 c$ refinement of OKIS (SUP 53898), although none of those final values was reported as negative. It is almost certainly due to systematic errors, perhaps related to absorption or to the twinning problem noted by OKIS. (2) Probably for the same reason, four reflections $(0,0,18,0,0,24,0,0,30$ and $0,0,36)$ were statistical outliers, all with $F_{o}>\left|F_{c}\right|$; they were given reduced weights by OKIS and weights of 0.0 by me (but were included in $R$ ). (3) There were moderately strong indications that $\mathrm{P}$ atoms are concentrated in the $\operatorname{Si}(\mathrm{P})(3)$ sites: the $U_{i i}$ 's are noticeably smaller (Table 2) and refinements of the distribution parameters continually suggested more electrons here than in the other two $\mathrm{Si}(\mathrm{P})$ sites. However, differences between the final distribution parameters were not reliable in a statistical sense and the final results are based on the same assumed distributions $(0.79 \mathrm{Si}$, $0.21 \mathrm{P}$ ) in all three sites.

Although the coordinate changes from the $R 3 c$ to the $R \overline{3} c$ structure are small in an absolute sense $0.1 \AA$ is the largest - they are, statistically, highly significant (the coordinate e.s.d.'s are $0.002 \AA$ or less). The general description of the structure is unchanged. 
OKIS note that the structure is 'isomorphous' with that of $\mathrm{Pd}_{8} \mathrm{Sb}_{3}$, which was also described in $R 3 c[a=$ 7.6152 (7), $c=43.032$ (7) $\AA$; Wopersnow \& Schubert (1976)]. Once again, refinement in $R \overline{3} c$ is to be preferred, leading to an $R$ of 0.126 for $533 F_{o}$ values (recovered directly from the published paper) compared to an $R$ of 0.14 reported for $R 3 c$. The $R \overline{3} c$ coordinates are given in Table 3 and the $U_{i j}$ 's in Table 4 . As in the case of perryite, these $R \overline{3} c$ coordinates differ from the $R 3 c$ coordinates by amounts which, though small (never more than $0.07 \AA$ ), are of great statistical significance in view of e.s.d.'s of $0.007 \AA$ or less.

\section{2. $\operatorname{Pt}(\mathrm{NCO})_{2}\left(\mathrm{C}_{12} \mathrm{H}_{12} \mathrm{~N}_{2}\right)$}

The structure of this compound, (4,4'-dimethyl$2,2^{\prime}$-bipyridyl- $N, N^{\prime}$ )bis(isocyanato)platinum(II), was described and refined in space group $C m c 2_{1}[a=$ 18.722 (6), $b=11.889$ (5), $\quad c=6.688$ (5) $\AA, \quad Z=4$; Coyer, Herber \& Cohen (1991); hereinafter referred to as $\mathrm{CHC}$. The resulting molecule was closely planar, no atom deviating from the plane $z=0.37$ by more than $0.13 \AA$; exact planarity leads immediately to the centrosymmetric space group $\mathrm{Cmcm}$. Refinement in $\mathrm{Cmcm}$, based on the 735 reflections [with $F_{o} \geq 6 \sigma\left(F_{o}\right) ; \mathrm{CHC}$ ] recovered from SUP 53843, converged routinely at $R=0.049$, compared to the 0.052 reported for $C m c 2_{1}$. The $\mathrm{Cmcm}$ refinement entailed 66 parameters, including $U_{i j}$ 's for all the heavier atoms and an isotropic secondary-extinction pai imeter [final value $0.14(1) \times 10^{-6}$ ]; the $\mathrm{H}$ atoms were placed in calculated positions (as in $\mathrm{CHC}$ ), with those on the $-\mathrm{CH}_{3}$ group disordered between two sets of half-occupied sites. Final heavy-atom coordinates are given in Table 5.

Changes from the earlier $C m c 2_{1}$ model involve only the out-of-plane $(z)$ coordinates and hence have little effect on the bond lengths and angles.

\section{1,3-Propanediammonium bis(dihydrogenmono- phosphate)}

The structure of this compound, $\left[\left(\mathrm{NH}_{3}\right)\left(\mathrm{CH}_{2}\right)_{3}\right.$ $\left.\left(\mathrm{NH}_{3}\right)\right]^{2+} .2\left(\mathrm{H}_{2} \mathrm{PO}_{4}\right)^{-}$, was refined in space group $C c$ [monoclinic, $a=18.543(3), \quad b=4.561$ (4), $\quad c=$ $15.342(3) \AA, \quad \beta=129.14(1)^{\circ}, \quad Z=4$; Kamoun, Jouini, Daoud, Durif \& Guitel (1992)]. It is better described in $C 2 / c$, with the propanediammonium cation lying on a twofold axis. Starting parameters were obtained by suitable symmetrizing and averaging of the coordinates in Table 1 of Kamoun et al. (1992) [after inversion of the signs of all three coordinates of $\mathrm{O}(13)$ and the $y$ coordinate of $\mathrm{O}(14)$ because of apparent misprints]. Least-squares refinement in $C 2 / c$, based on 3362 reflections recovered from SUP 54431 , led to an $R$ of 0.0254 for 102 parameters -
Table 3. $\mathrm{Pd}_{8} \mathrm{Sb}_{3}$ coordinates, space group $R \overline{3} c$

$x, y, z$ and $U_{\text {eq }}$ are multiplied by $10^{4}$.

$$
U_{\mathrm{eq}}=\frac{1}{3} \sum_{i} \sum_{j} U_{i j} a_{i}^{*} a_{j}^{*} \mathbf{a}_{i} \cdot \mathbf{a}_{j} .
$$

$\begin{array}{cccl}x & y & z & U_{\text {eq }}\left(\AA^{2}\right) \\ 3321(6) & 4028(7) & 522(1) & 217(8) \\ 3404(6) & 310(7) & 175(1) & 208(9) \\ 0 & 0 & 2188(2) & 272(11) \\ 0 & 0 & 620(2) & 228(10) \\ -3189(7) & 0 & 2500 & 182(9) \\ 0 & 0 & 1570(1) & 191(9) \\ 0 & 0 & 0 & 198(12)\end{array}$

Table 4. $\mathrm{Pd}_{8} \mathrm{Sb}_{3}$ anisotropic coefficients $\left(\AA^{2}\right)$, space group $R \overline{3} c$

$U_{i j}$ values have been multiplied by $10^{4}$. The form of the displacement factor is $\exp \left[-2 \pi^{2}\left(U_{11} h^{2} a^{* 2}+U_{22} k^{2} b^{* 2}+U_{33} l^{2} c^{* 2}+\right.\right.$ $\left.\left.2 U_{12} h k a^{*} b^{*}+2 U_{13} h l a^{*} c^{*}+2 U_{23} k l b^{*} c^{*}\right)\right]$.

$$
\begin{array}{lllllll}
U_{11} & U_{22} & U_{33} & U_{12} & U_{13} & U_{23}
\end{array}
$$

$\begin{array}{lllllll}\operatorname{Pd}(1) & 172(19) & 230(21) & 207(17) & 69(18) & 17(15) & -21(15)\end{array}$

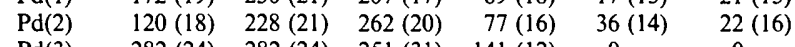

$\begin{array}{lllllll}\operatorname{Pd}(3) & 282(24) & 282(24) & 251(31) & 141(12) & 0 & 0\end{array}$

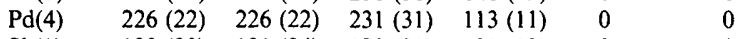

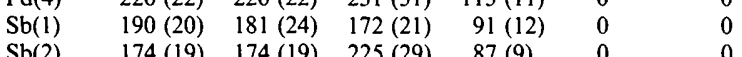

$\begin{array}{lllllll}\mathrm{Sb}(3) & 181(26) & 181(26) & 232(39) & 90(13) & 0 & 0\end{array}$

Table 5. $\operatorname{Pt}(\mathrm{NCO})_{2}\left(\mathrm{C}_{12} \mathrm{H}_{12} \mathrm{~N}_{2}\right)$ coordinates, space group $\mathrm{Cmcm}$

$x, y, z$ and $U_{\text {eq }}$ are multiplied by $10^{4}$.

$$
U_{\text {eq }}=\frac{1}{3} \sum_{i} \sum_{j} U_{i j} a_{i}^{*} a_{j}^{*} \mathbf{a}_{i} \cdot \mathbf{a}_{j} .
$$

$\begin{array}{lcccl} & x & y & z & U_{\text {eq }}\left(\AA^{2}\right) \\ \mathrm{Pt} & 0 & 5426.0(9) & 2500 & 339(2) \\ \mathrm{N}(1) & 693(7) & 4139(11) & 2500 & 360(30) \\ \mathrm{C}(1) & 1406(8) & 4237(14) & 2500 & 507(45) \\ \mathrm{C}(2) & 1840(9) & 3293(18) & 2500 & 626(51) \\ \mathrm{C}(3) & 1551(9) & 2227(17) & 2500 & 521(45) \\ \mathrm{C}(4) & 806(9) & 2140(14) & 2500 & 428(40) \\ \mathrm{C}(5) & 395(8) & 3099(14) & 2500 & 390(35) \\ \mathrm{C}(6) & 2015(10) & 1188(18) & 2500 & 755(57) \\ \mathrm{N}(2) & 748(10) & 6606(13) & 2500 & 636(49) \\ \mathrm{C}(7) & 999(9) & 7490(16) & 2500 & 492(45) \\ \mathrm{O} & 1297(9) & 8408(13) & 2500 & 920(50)\end{array}$

slightly better than the 0.027 reported by Kamoun et al. (1992) for, apparently, 200 parameters. As in the earlier $C c$ model, all atoms were included in the refinement, the $\mathrm{H}$ atoms with isotropic $B$ 's. Also included was an isotropic extinction coefficient [final value $15.0(5) \times 10^{-6}$ ]; it may have accounted for most of the improvement over the earlier refinement, as extinction was not taken into account by Kamoun et al. (1992). Coordinates are given in Table 6.

A noteworthy point in this example is that intensities were collected using $\operatorname{Ag} K \alpha$ radiation out to $\theta=$ $35^{\circ}\left(\sin \theta / \lambda=1.02 \AA^{-1}\right)$; as a result, formal precisions in the results are excellent $-0.001 \AA$ or less for the heavier atoms, less than $0.02 \AA$ for the $\mathrm{H}$ atoms. Changes in the bond lengths resulting from the higher-symmetry refinement were far larger. The 
Table 6. 1,3-Propanediammonium bis(dihydrogenmonophosphate) coordinates, space group $C 2 / c$

$x, y$ and $z$ are multiplied by $10^{5}$ for the non- $\mathrm{H}$ atoms and by $10^{4}$ for the $\mathrm{H}$ atoms. $U_{\mathrm{eq}}$ is multiplied by $10^{5}$.

\begin{tabular}{lcccc}
\multicolumn{5}{c}{$U_{\text {eq }}=\frac{1}{3} \sum_{i} \sum_{j} U_{i j} a_{i}^{*} a_{j}^{*} \mathbf{a}_{i} \cdot \mathbf{a}_{j} \cdot$} \\
P & $x$ & $y$ & $z$ & $U_{\text {eq }}\left(\AA^{2}\right)$ \\
$\mathrm{O}(1)$ & $18871(1)$ & $9942(3)$ & $57576(1)$ & $1413(2)$ \\
$\mathrm{O}(2)$ & $26253(3)$ & $33521(10)$ & $62094(4)$ & $1908(3)$ \\
$\mathrm{O}(3)$ & $16810(3)$ & $-4853(11)$ & $46946(4)$ & $2216(3)$ \\
$\mathrm{O}(4)$ & $23090(4)$ & $-15107(11)$ & $66573(4)$ & $2486(4)$ \\
$\mathrm{N}$ & $9979(3)$ & $20263(14)$ & $54774(4)$ & $2779(3)$ \\
$\mathrm{C}(1)$ & $7016(4)$ & $60352(15)$ & $65601(5)$ & $2152(4)$ \\
$\mathrm{C}(2)$ & $3532(5)$ & $42021(16)$ & $70240(6)$ & $2339(4)$ \\
& 0 & $60737(23)$ & 75000 & $2288(5)$ \\
& & & & \\
$\mathrm{H}(\mathrm{O} 2)$ & $1947(10)$ & $71(36)$ & $4529(12)$ & $4.2(3)$ \\
$\mathrm{H}(\mathrm{O} 3)$ & $2340(11)$ & $-2890(36)$ & $6422(13)$ & $4.5(3)$ \\
$\mathrm{H}(\mathrm{N} a)$ & $1160(8)$ & $7048(27)$ & $7088(10)$ & $2.6(2)$ \\
$\mathrm{H}(\mathrm{N} b)$ & $278(8)$ & $7168(27)$ & $6013(10)$ & $2.6(2)$ \\
$\mathrm{H}(\mathrm{N} c)$ & $885(8)$ & $4887(29)$ & $6305(9)$ & $2.7(2)$ \\
$\mathrm{H}(\mathrm{C} 1 a)$ & $871(8)$ & $2981(29)$ & $7597(11)$ & $3.2(3)$ \\
$\mathrm{H}(\mathrm{C} 1 b)$ & $-114(9)$ & $2946(29)$ & $6433(11)$ & $3.6(3)$ \\
$\mathrm{H}(\mathrm{C} 2)$ & $500(9)$ & $7378(28)$ & $8119(11)$ & $3.5(3)$
\end{tabular}

largest change was in the $\mathrm{N}-\mathrm{C}$ bond lengths: whereas the $C c$ refinement resulted in two highly disparate distances, at $1.522(3)$ and $1.455(2) \AA$, the revised structure shows the bonds to be equal, by symmetry, at 1.484 (1) $\AA$. Revised bond lengths and angles are given in Table 7 . The relatively large difference between the $\mathrm{P}=\mathrm{O}(1)$ and $\mathrm{P}=\mathrm{O}(4)$ bond lengths correlates well with the hydrogen bonding: $O(1)$ accepts two strong $\mathrm{O}-\mathrm{H} \cdots \mathrm{O}$ bonds $(\sim 2.61 \AA)$ plus a weaker $(3.04 \AA) \mathrm{N}-\mathrm{H} \cdots \mathrm{O}$ bond, while $\mathrm{O}(4)$ accepts two intermediate $(\sim 2.8 \AA) \mathrm{N}-\mathrm{H} \cdots \mathrm{O}$ bonds.

\section{1,2,7a-Trihydroxy-2-methylperhydro-1-phospha- indene 1-oxide}

This is probably the most interesting - and surely the most controversial - of the present examples. Crystals of this compound, $\mathrm{C}_{9} \mathrm{H}_{17} \mathrm{O}_{4} \mathrm{P}$, were described as monoclinic $\quad[a=12.385(4), \quad b=6.590(3), \quad c=$ 13.394 (4) $\AA, \beta=98.00(3)^{\circ}, Z=4$, space group $P 2_{1}$; Bartczak \& Yagbasan (1991); hereinafter referred to as BY]. The authors noted that the two molecules in the asymmetric unit 'have almost identical but inverted conformations'; the point of inversion relating the two molecules is so positioned as to generate, almost exactly, space group $P 2_{1} / n$. Indications of the near-singularities that must have arisen during the refinement in $P 2_{1}$ are some relatively large differences between equivalent bond lengths in the two molecules, ranging up to 0.18 (3) $\AA$. Further refinement in $P 2_{1} / n$ was clearly called for.

SUP 53907 included a listing of $2228 F_{o}$ and $F_{c}$ values - apparently the complete set of reflections
Table 7. 1,3-Propanediammonium bis(dihydrogenmonophosphate) bond lengths and angles, space group $C 2 / c$

$\begin{array}{lclc} & \text { Distance }(\AA) & \text { Distance }(\AA) \\ \mathrm{P}-\mathrm{O}(1) & 1.521(1) & \mathrm{O}(3)-\mathrm{H}(\mathrm{O} 3) & 0.747(19) \\ \mathrm{P}-\mathrm{O}(2) & 1.573(1) & \mathrm{N}-\mathrm{H}(\mathrm{N} a) & 0.850(14) \\ \mathrm{P}-\mathrm{O}(3) & 1.568(1) & \mathrm{N}-\mathrm{H}(\mathrm{N} b) & 0.869(14) \\ \mathrm{P}-\mathrm{O}(4) & 1.493(1) & \mathrm{N}-\mathrm{H}(\mathrm{N} c) & 0.844(14) \\ \mathrm{N}-\mathrm{C}(1) & 1.484(1) & \mathrm{C}(1)-\mathrm{H}(\mathrm{Cl} a) & 0.969(15) \\ \mathrm{C}(1)-\mathrm{C}(2) & 1.515(1) & \mathrm{C}(1)-\mathrm{H}(\mathrm{Cl} b) & 0.954(16) \\ \mathrm{O}(2)-\mathrm{H}(\mathrm{O} 2) & 0.729(18) & \mathrm{C}(2)-\mathrm{H}(\mathrm{C} 2) & 1.002(15) \\ & & & \\ & \text { Angle ( }) & & \text { Angle }\left(^{\circ}\right) \\ \mathrm{O}(2)-\mathrm{P}-\mathrm{O}(1) & 109.61(3) & \mathrm{H}(\mathrm{N} c)-\mathrm{N}-\mathrm{C}(1) & 107.4(10) \\ \mathrm{O}(3)-\mathrm{P}-\mathrm{O}(1) & 107.98(3) & \mathrm{H}(\mathrm{N} b)-\mathrm{N}-\mathrm{H}(\mathrm{N} a) & 110.3(13) \\ \mathrm{O}(4)-\mathrm{P}-\mathrm{O}(1) & 114.89(3) & \mathrm{H}(\mathrm{N} c)-\mathrm{N}-\mathrm{H}(\mathrm{N} a) & 109.0(13) \\ \mathrm{O}(3)-\mathrm{P}-\mathrm{O}(2) & 105.06(3) & \mathrm{H}(\mathrm{N} c)-\mathrm{N}-\mathrm{H}(\mathrm{N} b) & 107.8(13) \\ \mathrm{O}(4)-\mathrm{P}-\mathrm{O}(2) & 109.58(3) & \mathrm{H}(\mathrm{Cl} a)-\mathrm{C}(1)-\mathrm{N} & 106.1(9) \\ \mathrm{O}(4)-\mathrm{P}-\mathrm{O}(3) & 109.25(3) & \mathrm{H}(\mathrm{Cl} b)-\mathrm{C}(1)-\mathrm{N} & 106.9(9) \\ \mathrm{C}(2)-\mathrm{C}(1)-\mathrm{N} & 111.42(7) & \mathrm{H}(\mathrm{Cl} a)-\mathrm{C}(1)-\mathrm{C}(2) & 111.7(9) \\ \mathrm{C}(1)-\mathrm{C}(2)-\mathrm{C}(1) & 111.40(8) & \mathrm{H}(\mathrm{C} 1 b)-\mathrm{C}(1)-\mathrm{C}(2) & 112.8(9) \\ \mathrm{H}(\mathrm{O} 2)-\mathrm{O}(2)-\mathrm{P} & 115.9(14) & \mathrm{H}(\mathrm{Cl} b)-\mathrm{C}(1)-\mathrm{H}(\mathrm{Cl} a) & 107.6(13) \\ \mathrm{H}(\mathrm{O} 3)-\mathrm{O}(3)-\mathrm{P} & 109.7(14) & \mathrm{H}(\mathrm{C} 2)-\mathrm{C}(2)-\mathrm{C}(1) & 111.6(9) \\ \mathrm{H}(\mathrm{N} a)-\mathrm{N}-\mathrm{C}(1) & 109.0(9) & \mathrm{H}(\mathrm{C} 2)-\mathrm{C}(2)-\mathrm{C}\left(1^{\prime}\right) & 107.5(9) \\ \mathrm{H}(\mathrm{N} b)-\mathrm{N}-\mathrm{C}(1) & 113.3(9) & \mathrm{H}(\mathrm{C} 2)-\mathrm{C}(2)-\mathrm{H}\left(\mathrm{C} 2^{\prime}\right) & 107.2(12) \\ & & & \end{array}$

within the quadrant surveyed $\left(\theta_{\max }=70^{\circ}\right.$ for $\mathrm{Cu} K \alpha$ radiation; BY). All $F_{o}$ values were positive. Included were 192 of the type $(h 0 l)$ with $(h+l)$ odd, expected to be missing if the true space group is $P 2_{1} / n$; these showed $F_{o}$ 's ranging up to $7.8 \mathrm{e}^{-}$, compared to a maximum $F_{o}$ of $119.3 \mathrm{e}^{-}$(for the $\overline{202}$ reflection). While values of $\sigma_{F}$ were not included, I estimate that, on the average, the cutoff criterion $I>3 \sigma(I)$ used by BY in their analysis of the structure lies at about $F_{o}=1.7 \mathrm{e}^{-} ; 27$ of the 'odd' $h 0 l$ 's exceeded that value. On the face of it, then, $P 2_{1} / n$ should be immediately rejected; but more of this later.

Refinement in $P 2_{1} / n$ proceeded normally. Starting coordinates were from Table 1 of BY, appropriately symmetrized and averaged; full-matrix least-squares adjustment of 196 parameters (coordinates for all atoms; anisotropic $U_{i j}$ 's for $\mathrm{P}, \mathrm{O}$ and $\mathrm{C}$ atoms; isotropic $B$ 's for $\mathrm{H}$ atoms; scale and extinction coefficients) quickly led to convergence (maximum shift $/ \sigma 0.05$ ) at $R=0.045$ for 2036 reflections - all except the 'odd' $h 0 l$ 's; if the latter are included, with $F_{c}=0, R$ becomes 0.054 for the entire list of 2228 reflections. For the $P 2_{1}$ model, BY report an $R$ of 0.044 for 1902 reflections with $I>3 \sigma(I)$; for the $P 2_{1} / n$ model, $R$ is 0.045 for 1822 reflections with $F>1.7 \mathrm{e}^{-}$. Final $P 2{ }_{1} / n$ coordinates are given in Table 8 .

At this stage, then, we face an apparent dilemma: on the one hand, the $P 2_{1} / n$ model, with approximately half as many parameters, yields essentially the same agreement index as the $P 2_{1}$ model and hence should be preferred; moreover, the resulting bond lengths are more normal. [For example, whereas the $P 2_{1}$ coordinates lead to $\mathrm{C}(5)-\mathrm{C}(6)$ bond lengths of 
Table $8 . \mathrm{C}_{9} \mathrm{H}_{17} \mathrm{O}_{4} \mathrm{P}$ coordinates, space group $P 2_{1} / n$ $x, y, z$ and $U_{\text {eq }}$ are multiplied by $10^{4} . B$ is the isotropic displacement parameter

\begin{tabular}{lcccc}
\multicolumn{5}{c}{$U_{\text {eq }}=\frac{1}{3} \sum_{i} \sum_{j} U_{i j} a_{i}{ }^{*} a_{j}{ }^{*} \mathbf{a}_{i} \cdot \mathbf{a}_{j}$} \\
& $x$ & $y$ & $z$ & $U_{\text {eq }}\left(\AA^{2}\right)$ \\
$\mathrm{P}$ & & & & $285(1)$ \\
$\mathrm{O}(1)$ & $7325.9(4)$ & $34.1(9)$ & $2751.9(4)$ & $287(4)$ \\
$\mathrm{O}(2)$ & $6966(2)$ & $1326(3)$ & $3607(1)$ & $401(4)$ \\
$\mathrm{O}(3)$ & $5930(2)$ & $-3939(3)$ & $2890(2)$ & $453(5)$ \\
$\mathrm{O}(4)$ & $4693(2)$ & $2506(3)$ & $2970(2)$ & $376(4)$ \\
$\mathrm{C}(1)$ & $5698(2)$ & $-2086(4)$ & $3385(2)$ & $333(5)$ \\
$\mathrm{C}(2)$ & $4472(2)$ & $-1717(4)$ & $3084(2)$ & $374(6)$ \\
$\mathrm{C}(3)$ & $4285(2)$ & $-594(4)$ & $2085(2)$ & $332(5)$ \\
$\mathrm{C}(4)$ & $3125(2)$ & $81(5)$ & $1707(2)$ & $446(6)$ \\
$\mathrm{C}(5)$ & $3080(2)$ & $1251(6)$ & $726(2)$ & $546(8)$ \\
$\mathrm{C}(6)$ & $3867(2)$ & $3049(5)$ & $821(3)$ & $550(8)$ \\
$\mathrm{C}(7)$ & $5036(2)$ & $2390(5)$ & $1218(2)$ & $413(6)$ \\
$\mathrm{C}(8)$ & $5055(2)$ & $1271(3)$ & $2206(2)$ & $291(5)$ \\
$\mathrm{C}(9)$ & $6064(3)$ & $-2216(6)$ & $4500(3)$ & $541(8)$ \\
& & & & \\
& & & & \\
$\mathrm{H}(\mathrm{O} 2)$ & $7387(29)$ & $2218(62)$ & $3418(27)$ & $7.0(10)$ \\
$\mathrm{H}(\mathrm{O} 3)$ & $6612(33)$ & $-4152(65)$ & $3101(29)$ & $8.1(12)$ \\
$\mathrm{H}(\mathrm{O} 4)$ & $5038(33)$ & $3311(66)$ & $3028(32)$ & $7.7(13)$ \\
$\mathrm{H}(2 a)$ & $4207(22)$ & $-990(44)$ & $3598(20)$ & $3.7(6)$ \\
$\mathrm{H}(2 b)$ & $4082(25)$ & $-2926(52)$ & $3039(22)$ & $4.8(7)$ \\
$\mathrm{H}(3)$ & $4553(20)$ & $-1474(41)$ & $1612(19)$ & $3.0(5)$ \\
$\mathrm{H}(4 a)$ & $2895(20)$ & $840(43)$ & $2289(20)$ & $3.5(6)$ \\
$\mathrm{H}(4 b)$ & $2665(23)$ & $-1106(48)$ & $1615(21)$ & $4.3(7)$ \\
$\mathrm{H}(5 a)$ & $2331(27)$ & $1759(52)$ & $506(24)$ & $5.6(8)$ \\
$\mathrm{H}(5 b)$ & $3249(24)$ & $356(52)$ & $136(23)$ & $5.2(8)$ \\
$\mathrm{H}(6 a)$ & $3644(25)$ & $4021(52)$ & $1384(25)$ & $5.5(8)$ \\
$\mathrm{H}(6 b)$ & $3865(25)$ & $3536(51)$ & $183(24)$ & $5.1(8)$ \\
$\mathrm{H}(7 a)$ & $5574(25)$ & $3513(52)$ & $1364(23)$ & $5.3(8)$ \\
$\mathrm{H}(7 b)$ & $5306(21)$ & $1455(44)$ & $777(19)$ & $3.4(6)$ \\
$\mathrm{H}(9 a)$ & $6897(28)$ & $-2507(55)$ & $4638(24)$ & $6.0(8)$ \\
$\mathrm{H}(9 b)$ & $5943(30)$ & $-991(62)$ & $4810(27)$ & $6.6(10)$ \\
$\mathrm{H}(9 c)$ & $5689(28)$ & $-3122(61)$ & $4791(27)$ & $6.4(9)$
\end{tabular}

1.44 (2) $\AA$ in one molecule and $1.62(2) \AA$ in the other, the $P 2_{1} / n$ structure has the two distances equal by symmetry at $1.528(5) \AA$.] On the other hand, the appearance of the 'odd' $h 0 l$ 's argues strongly in favor of $P 2_{1}$. What should we conclude?

Fortunately, in this case there is one fairly large body of evidence that we can turn to - the weak reflections. As has been pointed out on numerous occasions (e.g. Dunitz, 1979; Marsh, 1981; Schomaker \& Marsh, 1979), the weak reflections are the ones most sensitive to the centrosymmetric-noncentrosymmetric ambiguity since the antisymmetric distortion present in a noncentrosymmetric model adds an out-of-phase ' $B$ ' component of the structure factors, which becomes significant when the ' $A$ ' value - and hence $|F|$ itself - is small. In the present case, the supplementary material contains $F_{o}$ values for 235 reflections (excluding the 'odd' $h 0 l$ 's) with $F<1.7 \mathrm{e}^{-}$[the estimated $I>3 \sigma(I)$ cut-off]. For these 235 reflections, I compared $F_{o}$ with the $F_{c}$ 's obtained from the final $P 2_{1}(\mathrm{BY})$ and $P 2_{1} / n$ (Table 8) models; the results are summarized in Table 9 . They seem unambiguous in favoring $P 2_{1} / n$ over $P 2_{1}$ : not only is $R$ much smaller but the average value of $\left|F_{c}\right|$ is much closer to what one would expect.
Table 9. $\mathrm{C}_{9} \mathrm{H}_{17} \mathrm{O}_{4} \mathrm{P}$ : comparisons between $F_{o}$ and $F_{c}$ for the 235 reflections (excluding 'odd' hol's) with $F_{o}<1.7 \mathrm{e}^{-}$

The $F_{c}$ values for the $P 2_{1}$ structure are from SUP 53907, for the $P 2_{1} / n$ structure from the present refinement.

$\begin{array}{lcc} & P 2_{1} & P 2_{1} / n \\ \sum_{\sum_{o}}\left|F_{c}\right| & 233.9 & 233.9 \\ \sum_{R}|\Delta F| & 385.9 & 198.0 \\ & 195.6 & 103.0 \\ & 0.84 & 0.44\end{array}$

If the arguments in favor of $P 2_{1} / n$ are to be persuasive, we must explain the apparent presence of reflections $h 0 l$ with $(h+l)$ odd - and in particular the 27 with $F_{o}>1.7 \mathrm{e}^{-}$. Of these 27 , all except three (among the weakest) are of the type with $h$ odd and $l$ even (and small), rather than the other way around. This trend - of $F_{o}$ being larger than $\left|F_{c}\right|$ for weak reflections with $l$ even and small - seems to pervade the entire reflection list. Was a small twin component present?*

So here we have a real quandary: do we accept the axiom, taught by rote to all crystallographers, that systematic absences cannot be violated, and thus the space group is surely $P 2_{1}$ ? Or do we accept a different and probably larger body of evidence - the improved agreement for the weak reflections and the more reasonable structural results - that argues in favor of $P 2_{1} / n$ ?

\section{Concluding remarks}

These examples point up, once again, the insensitivity of diffraction experiments to the decision as to whether a crystal structure is centrosymmetric or only approximately so. In all these instances, apparently satisfactory agreement between observed and calculated intensities had been obtained by the original authors on the basis of noncentrosymmetric models, yet equally satisfactory refinement - and, in most cases, more reasonable bond lengths - can be obtained in the corresponding centrosymmetric space groups. Even in the final example, where apparent violations of systematic absences should provide clear proof of the noncentrosymmetric space group, other evidence suggests that the decision is not clearcut. What should one do? It continues to be my position that, barring conclusive evidence to the contrary, one should opt for the centrosymmetric

\footnotetext{
* Among many helpful referees' comments was the logical suggestion that the appearance of 'forbidden' reflections might be due to multiple reflection (the 'Renninger' effect) or to $\lambda / 2$ wavelength contamination.
} 
description, with the implicit admission that diffraction data may well be unable to provide an unambiguous result.*

* Lists of structure factors for all five compounds, $U_{i j}$ 's for compounds (2), (3) and (4) and assumed $\mathrm{H}$-atom coordinates for compound (2) have been deposited with the IUCr (Reference: BU311). Copies may be obtained through The Managing Editor, International Union of Crystallography, 5 Abbey Square, Chester CHI 2HU, England.

References

Bartczak, T. J. \& Yagbasan, R. (1991). Acta Cryst. C47, $1750-1752$.
Coyer, M., Herber, R. H. \& Cohen, S. (1991). Acta Cryst. C47, 1376-1378.

DuniTZ, J. D. (1979). X-ray Analysis and the Structure of Organic Molecules, pp. 208-209. Ithaca and London: Cornell Univ. Press.

ERmer, O. \& Dunitz, J. D. (1970). Acta Cryst. A26, 163.

Kamoun, S., Jouin, A., Daoud, A., Durif, A. \& Guitel, J. C. (1992). Acta Cryst. C48, 133-135.

MARSH, R. E. (1981). Acta Cryst. B37, 1985-1988.

MARSH, R. E. (1986). Acta Cryst. B42, 193-198.

Okada, A., Kobayashi, K., Ito, T. \& Sakurai, T. (1991). Acta Cryst. C47, 1358-1361.

Schomaker, V. \& MARsh, R. E. (1979). Acta Cryst. B35, 23312336.

Wopersnow, W. \& Schubert, K. (1976). J. Less-Common Met. 48, 79-87.

Acta Cryst. (1994). A50, 455-461

\title{
Strip-Projection Approach to a New Model of the AlMnSi Icosahedral Quasicrystal
}

\author{
BY Y. F. ChENG* AND J. GJøNNES \\ Department of Physics and Center for Materials Research, University of Oslo, PO Box 1048 Blindern, \\ 0316 Oslo, Norway
}

(Received 24 June 1992; accepted 17 November 1993)

\begin{abstract}
Recently, an approach to the atomic model of the AlMnSi quascrystalline phase has been proposed. Rhombohedra that differ from those used in the Penrose tiling were used to form an approximant. It gave a good approximation to the AlMnSi quasicrystal structure. In the present paper, a strip-projection approach to this model is implemented. It is demonstrated that the tiling, with the tile edges along the threefold axes of the icosahedral symmetry, can also be used to describe the structure of the AlMnSi icosahedral quasicrystal. The Mackay icosahedra are decorated at the vertices of the tiles.
\end{abstract}

\section{Introduction}

After the first discovery of an icosahedral quasicrystal (Shechtman, Blech, Gratias \& Cahn, 1984), various models were proposed to describe its structure (Elser \& Henley, 1985; Guyot \& Audier, 1985; Yang \& Kuo, 1986; Cahn, Gratias \& Mozer, 1988a; Duneau \& Oguey, 1989; Pan, Cheng \& Li, 1990; Andersson, Lidin, Jacob \& Terasaki, 1991). A generally accepted geometrical model for describing

\footnotetext{
*Present address: Institute of Physics, Chinese Academy of Sciences, PO Box 603, Beijing 100080, People's Republic of China.
}

the quasiperiodicity and the symmetry of the quasicrystals has been the Penrose tiling (Penrose, 1974; Mackay, 1982). It consists of two different rohombohedra [a prolate rhombohedron (PR) and an oblate rhombohedron (OR)] as the building tiles, packed according to a special matching rule. However, the determination of atomic structure remains a principal problem. One of the approaches is to decorate atoms in the two kinds of Penrose tiles. An example shown by Elser \& Henley (1985) is that the body-centred cubic (b.c.c.) structure of the crystalline approximant $\alpha$-AlMnSi phase was decomposed into a periodic packing of the $\mathrm{PR}$ and $\mathrm{OR}$, and its atomic decoration was proposed to be included in the icosahedral phase. Some models were proposed based on this approach (Henley \& Elser, 1986; Guyot \& Audier, 1985).

A different viewpoint was offered by Audier \& Guyot (1988). From the similarity of intensity distribution in the diffraction patterns of both the $\alpha-\mathrm{AlMnSi}$ phase and the quasicrystalline phase, they suggested that the Mackay icosahedron, which is a 54-atom cluster [(AlSi $)_{42} \mathrm{Mn}_{12}$ ] with icosahedral symmetry, is a common building block in both phases. Comparison of Patterson syntheses for both the phases (Cahn, Gratias \& Mozer, 1988b) lends support to this assumption, i.e. a description of the AlMnSi quasicrystal as a packing of Mackay 